\title{
Echocardiographic measurement of epicardial adipose tissue thickness in patients with microvascular angina
}

\author{
MACITT KALÇIK ${ }^{1, *}$, MAHMUT YESIN ${ }^{2}$, AHMET GÜNER $^{3}$, EMRAH BAYAM $^{4}$, \\ MUCAHİT YETIM ${ }^{1}$, TOLGA DOĞAN ${ }^{1}$, LÜTFÜ BEKAR ${ }^{1}$, OĞUZHAN ÇELIK ${ }^{5}$, \\ YUSUF KARAVELIOĞLU ${ }^{1}$
}

\author{
${ }^{1}$ Faculty of Medicine, Department of Cardiology, Hitit University, Corum, Turkey \\ ${ }^{2}$ Department of Cardiology, Kars Harakani State Hospital, Kars, Turkey \\ ${ }^{3}$ Department of Cardiology, Kosuyolu Kartal Training and Research Hopital, Istanbul, Turkey \\ ${ }^{4}$ Department of Cardiology, Umraniye Training and Research Hospital, Istanbul, Turkey \\ ${ }^{5}$ Department of Cardiology, Mugla Sitki Koçman University Training and Research Hospital, Mugla, Turkey \\ ${ }^{*}$ Corresponding author: Macit Kalçk, MD; Faculty of Medicine, Department of Cardiology, Hitit University, Buharaevler Mah. Buhara 25. Sok. \\ No:1/A, Daire: 22, Çorum, Turkey; Phone: +90 53649217 89; Fax: +90 36451178 89; E-mail: macitkalcik@yahoo.com
}

(Received: April 3, 2019; Revised manuscript received: April 12, 2019; Accepted: April 16, 2019)

\begin{abstract}
Introduction: Impaired coronary microcirculation, inflammation, and endothelial dysfunction were reported etiological factors for microvascular angina (MVA). Recently, increased epicardial adipose tissue (EAT) thickness has been associated with hypertension, metabolic syndrome, and coronary artery disease in general population. In this study, we aimed to evaluate the EAT thickness in patients with MVA. Methods: This study enrolled 200 patients ( 83 males; mean age: $55.4 \pm 8.2$ years) who have been diagnosed with MVA and 200 controls ( 89 males; mean age: $54.4 \pm 8.5$ years). All patients underwent transthoracic echocardiography, and EAT thickness was measured from a parasternal long-axis view as the hypoechoic space on the right ventricular free wall. Results: The mean EAT thickness was significantly higher in MVA patients than the controls $(5.5 \pm 1.1$ vs. $4.9 \pm 0.7 \mathrm{~mm} ; p<0.001)$. Multiple logistic regression analysis showed that increased EAT thickness was an independent predictor of MVA $(\mathrm{OR}=1.183,95 \% \mathrm{CI}=1.063-1.489 ; p=0.023)$. In receiver operating characteristic curve analyses, EAT thickness above $5.3 \mathrm{~mm}$ predicted MVA with a sentivity of $68 \%$ and a specificity of $63 \%$ ( $\mathrm{AUC}=0.711,95 \% \mathrm{CI}=0.659-0.762 ; p<0.001)$. Conclusions: The EAT thickness was observed significantly higher in MVA patients as compared to controls. Increased EAT thickness may be associated with mechanisms that play a major role in the pathogenesis of MVA.
\end{abstract}

Keywords: coronary angiography, coronary artery disease, echocardiography, epicardial adipose tissue thickness, microvascular angina

\section{Introduction}

Coronary angiography may reveal normal coronary arteries in a significant proportion $(20 \%-30 \%)$ of patients presenting with typical angina pectoris [1]. Although non-cardiac causes may be responsible for the chest pain, a considerable subset of these patients have true angina due to myocardial ischemia in the absence of angiographically significant coronary stenosis [2]. The term microvascular angina (MVA) is used to describe patients with typical angina pectoris and a positive stress test (classic downsloping ST segment depression on treadmill exercise test, and/or a reversible perfusion defect on radionuclear myocardial perfusion scan), in the absence of significant coronary stenosis on angiography and other cardiac diseases [3]. Coronary microvascular dysfunction was suggested to be the underlying pathophysiologic mechanism. Moreover, endothelial dysfunction with subsequent microvascular ischemia has been implicated as an important contributing factor [4]. Although several studies reported that patients with MVA supposed to have excellent long-term clinical outcome [5], other clinical reports have been reported that

This is an open-access article distributed under the terms of the Creative Commons Attribution-NonCommercial 4.0 International License, which permits unrestricted use, distribution, and reproduction in any medium for non-commercial purposes, provided the original author and source are credited, a link to the CC License is provided, and changes - if any - are indicated. 
a considerable percentage of those patients might have a higher risk of unfavorable clinical outcomes [6].

Epicardial adipose tissue (EAT) is a true visceral fat tissue, deposited in proximity to the atrium, the right ventricle's free wall, and left ventricular apex of the heart [7]. Under normal conditions, EAT has important functions, such as protecting vascular functions and providing energy requirement of the heart by producing free fatty acids, which are energy sources for myocardium. However, when volume of the EAT increases, this tissue acquires a prothrombotic and proinflammatory characteristics [8]. Increased EAT thickness has been defined as a cardiometabolic risk factor with a possible role in the pathogenesis of cardiovascular diseases, such as hypertension, insulin resistance, metabolic syndrome, and coronary artery disease [9]. However, the role of increased EAT thickness in patients with MVA has not been investigated yet.

In this study, we aimed to investigate the possible role of increased EAT thickness in the pathogenesis of MVA.

\section{Methods}

\section{Study population}

A total of 200 patients ( 83 males; mean age: $55.4 \pm 8.2$ years) who have been diagnosed with MVA and 200 controls (89 males; mean age: $54.4 \pm 8.5$ years) were enrolled in this single-center case-control study between May 2016 and January 2019. Patients with moderate to severe left ventricular systolic dysfunction, end-stage liver disease, renal insufficiency, chronic inflammatory diseases, connective tissue diseases, and malignancies were excluded from the study. Patients who had angina pectoris with detectable ischemia on non-invasive tests, such as treadmill stress test or myocardial perfusion scintigraphy and without any evidence of stenosis or vasospasm of epicardial coronary arteries during invasive coronary angiography were diagnosed as MVA. Age- and sex-matched asymptomatic controls without any detectable ischemia on non-invasive tests constituted the control group. All patients underwent transthoracic echocardiography (TTE). Complete blood count and blood chemistry panel were measured in all patients during admission. All demographic, laboratory, and echocardiographic parameters were recorded into a data set and compared between MVA patients and controls. All patients provided a written informed consent and the study protocol was approved by the local ethics committee of the Kosuyolu Kartal Training and Research Hospital in accordance with the Declaration of Helsinki and Good Clinical Practice guidelines.

\section{Laboratory analysis}

In order to perform complete blood count and blood chemistry panel, venous blood samples were collected after $12 \mathrm{~h}$ of fasting by a clean puncture of an antecubital vein from all patients. Complete blood countings were measured on Sysmex XT2000i analyser (Sysmex Corporation, Kobe, Japan). Fasting blood glucose, blood urea nitrogen, creatinine, uric acid, total bilirubin, alanine aminotransferase, aspartate aminotransferase, total cholesterol (TC), high-density lipoprotein (HDL), and triglyceride (TG) levels were also measured on an autoanalyzer (Siemens Advia 2400 Chemistry System, Siemens Diagnostic, Tarrytown, NY, USA). Low-density lipoprotein (LDL) was calculated using the Friedewald formula: $\mathrm{LDL}(\mathrm{mg} / \mathrm{dl})=\mathrm{TC}-(\mathrm{HDL}+\mathrm{TG} / 5)[10]$.

\section{Echocardiography}

All patients underwent TTE performed by the same cardiologist using Vivid 5 echocardiography device (GE Vingmed Ultrasound AS, Horten, Norway) and $3.2 \mathrm{mHz}$ adult probe with the patient in the left lateral decubitus position. In all patients, the left ventricular posterior wall thickness (PWT), interventricular septal thickness (IVST), left ventricular end-systolic diameter (LVESD), left ventricular end-diastolic diameter (LVEDD), and left atrial diameter (LAD) were measured on the parasternal long-axis view. The early diastolic peak flow velocity (E wave) and the late diastolic peak flow velocity (A wave) were measured using a color-guided pulsed Doppler echocardiographic examination. Left ventricular ejection fractions (LVEFs) of the patients were calculated using biplane Simpson's method.

\section{Measurement of EAT thickness}

The measurement of EAT thickness was performed by TTE from a parasternal long-axis view on the right ventricle's free wall at end-diastole, and the greatest perpendicular distance to the aortic annulus was achieved and averaged over three cardiac cycles [11]. In parasternal long-axis window, hypoechoic space on the right ventricular free wall was defined as EAT (Fig. 1).

\section{Statistical analyses}

Statistical analyses were performed using IBM SPSS Statistics for Windows, version 19.0 (IBM Corp., Armonk, NY, USA). Descriptive statistics were reported as mean \pm standard deviation for continuous variables with normal distribution or median (25th-75th percentiles) values for continuous variables without normal distribution and as frequency with percentages for the categorical variables. The Shapiro-Wilk and Kolmogorov-Smirnov tests were used to test the normality of the distribution of continuous variables. Categorical variables were 


\section{Kalçık et al.}

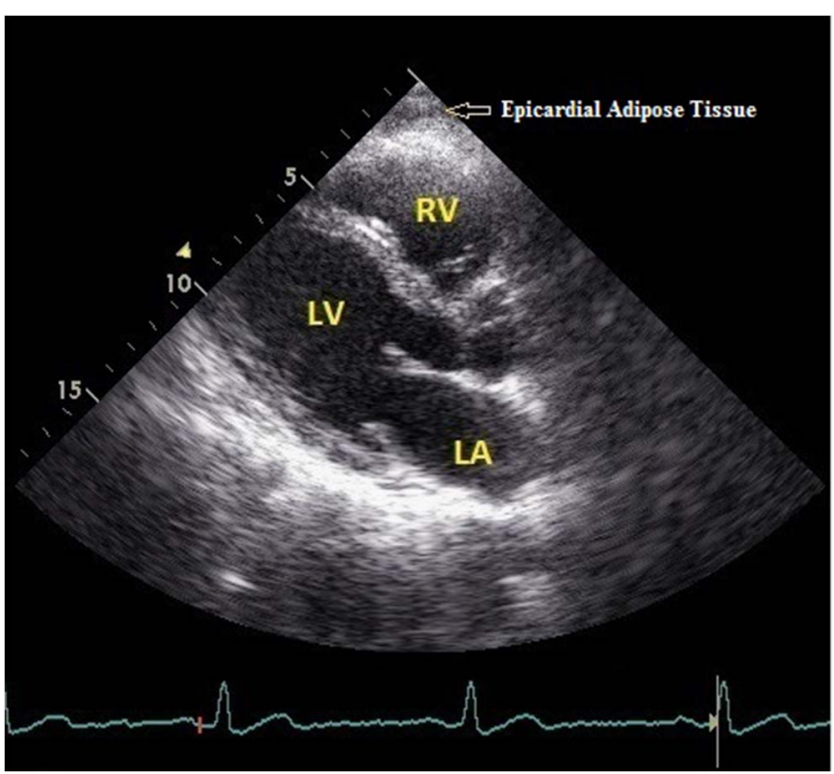

Fig. 1. Transthoracic echocardiography view revealing the epicardial adipose tissue on the the right ventricle's free wall from a parasternal long-axis view

compared with $\chi^{2}$ or Fisher's exact tests. Student's $t$-test or Mann-Whitney $U$ test was used to compare continuous variables as appropriate. The significance level was accepted as $p<0.05$ in all statistical analyses. A logistic regression analysis was performed in order to identify any independent associates of MVA. A receiver operating characteristic (ROC) curve analysis was performed to evaluate the sensitivity, specificity, area under the curve (AUC), and confidence interval (CI) of EAT for predicting MVA.

\section{Results}

Demographic, laboratory, and echocardiographic characteristics of the study population are presented in Table I. Age and gender distribution were similar between patients and controls. There was no significant difference in terms of systolic and diastolic blood pressure measurements and heart rate values between the groups. The prevalences of hypertension, diabetes mellitus, dyslipidemia, and smoking status were similar between the groups. Routine serum biomarkers, such as glucose, urea, creatinine, uric acid, bilirubin, alanine aminotransferase, aspartate aminotransferase, TC, HDL, LDL, TG, and complete blood count parameters, were similar between the patients and the controls. The echocardiographic parameters including LVEF, LVESD, LVEDD, LAD, IVST, PWT, and left atrial $\mathrm{E}$ and $\mathrm{A}$ waves were found to be similar between the groups. The mean EAT thickness was significantly higher in MVA patients than the controls $\quad(5.5 \pm 1.1$ vs. $4.9 \pm 0.7 \mathrm{~mm} ; p<0.001)$ (Fig. 2A).
The univariate parameters, which were possible predictors of MVA, were taken into multiple logistic regression analysis. Increased EAT thickness was an independent predictor of MVA (odds ratio $=1.183,95 \%$ $\mathrm{CI}=1.063-1.489 ; p=0.023$ ) (Table II). In the ROC curve analysis, EAT thickness above $5.3 \mathrm{~mm}$ predicted MVA with a sentivity of $69 \%$ and a specificity of $64 \%$ $(\mathrm{AUC}=0.711, \quad 95 \% \quad \mathrm{CI}=0.659-0.762 ; \quad p<0.001)$ (Fig. 2B).

\section{Discussion}

In this observational case-control study, we have focused on the role of increased EAT thickness in patients with MVA. The EAT thickness was observed significantly higher in MVA patients as compared to controls with similar cardiovascular risk factors. These results support that increased EAT thickness may be associated with mechanisms, which play a major role in the pathogenesis of MVA.

Epicardial coronary artery stenosis is usually responsible for myocardial ischemia. However, with the technical advancements in the past 30 years, studies have shown that abnormalities in coronary microcirculation may also cause myocardial ischemia in patients with normal coronary arteries [12]. In 1973, Kemp [13] first described these patients as having MVA, defined as typical angina pectoris with abnormal stress test results indicative of myocardial ischemia and normal coronary arteries on coronary angiography. Since the original description of cardiac syndrome $\mathrm{X}$ over 30 years ago, a large number of studies have focused on establishing an ischemic origin for this condition. The pathophysiology of MVA is multifactorial and endothelial dysfunction with subsequent microvascular ischemia has been implicated as an important contributing factor [4].

EAT located beneath the visceral pericardium is a particular variety of visceral fat tissue. The physiological, biochemical, and biomolecular properties of EAT and the possible paracrine reactions have been reported in previous studies [14]. Various studies have highlighted the potential importance of EAT in relation to inflammatory burden in cardiovascular diseases, such as coronary artery disease [15], subclinical atherosclerosis [16], and metabolic syndrome [11]. The EAT has been reported to have high capacity of local proinflammatory activity [17]. There is growing evidence that the changes in perivascular tissues surrounding epicardial coronary arteries could alter vascular homeostasis and contribute to endothelial dysfunction, amplification of vascular inflammation, intimal lesions, and plaque progression by an outside-to-inside signaling mechanism $[18,19]$.

The EAT is present over the base of the heart, the atrioventricular groove, bases of great vessels, along the distribution of coronary arteries, over the right 
Table I The comparison of demographic, echocardiographic, and laboratory parameters between patient and control groups

\begin{tabular}{|c|c|c|c|}
\hline & MVA $(n=200)$ & Controls $(n=200)$ & $p$ value \\
\hline \multicolumn{4}{|l|}{ Demographic parameters } \\
\hline Age (years) & $55.4 \pm 8.2$ & $54.4 \pm 8.5$ & 0.231 \\
\hline Gender: male $[n(\%)]$ & $83(41.5)$ & $89(44.5)$ & 0.545 \\
\hline BMI $\left(\mathrm{kg} / \mathrm{m}^{2}\right)$ & $29.3(25.1-31.5)$ & $28.9(26.2-31.8)$ & 0.874 \\
\hline $\mathrm{SBP}(\mathrm{mmHg})$ & $138.2 \pm 16.3$ & $134.5 \pm 11.4$ & 0.475 \\
\hline $\mathrm{DBP}(\mathrm{mmHg})$ & $83.6 \pm 9.3$ & $82.5 \pm 10.3$ & 0.674 \\
\hline Heart rate (beats/min) & $77.5 \pm 11.3$ & $74.6 \pm 10.4$ & 0.124 \\
\hline Hypertension $[n(\%)]$ & $66(33)$ & $54(27)$ & 0.190 \\
\hline Diabetes mellitus $[n(\%)]$ & $41(20.5)$ & $36(18)$ & 0.526 \\
\hline Dyslipidemia $[n(\%)]$ & $44(22)$ & $55(27.5)$ & 0.203 \\
\hline Smoking status $[n(\%)]$ & $50(25)$ & $46(23)$ & 0.640 \\
\hline \multicolumn{4}{|l|}{ Echocardiographic parameters } \\
\hline LVEF (\%) & $64.7 \pm 5.1$ & $65.6 \pm 4.3$ & 0.421 \\
\hline $\mathrm{LAD}(\mathrm{mm})$ & $38(35.7-40.2)$ & $38(36.7-40.5)$ & 0.579 \\
\hline $\operatorname{LVEDD}(\mathrm{cm})$ & $4.5(4.3-4.9)$ & $4.3(4.2-4.8)$ & 0.312 \\
\hline $\operatorname{LVESD}(\mathrm{cm})$ & $2.96 \pm 0.65$ & $2.93 \pm 0.53$ & 0.788 \\
\hline IVST (mm) & $11(11-12)$ & $11(11-12)$ & 0.178 \\
\hline PWT (mm) & $11(11-12)$ & $11(11-12)$ & 0.751 \\
\hline $\mathrm{E}(\mathrm{cm} / \mathrm{s})$ & $0.6(0.5-0.7)$ & $0.7(0.5-0.9)$ & 0.378 \\
\hline $\mathrm{A}(\mathrm{cm} / \mathrm{s})$ & $1(0.8-1)$ & $0.95(0.7-1)$ & 0.266 \\
\hline \multicolumn{4}{|l|}{ Laboratory parameters } \\
\hline Glucose $(\mathrm{mg} / \mathrm{dl})$ & $94.8 \pm 11.2$ & $96.3 \pm 9.8$ & 0.742 \\
\hline BUN (g/dl) & $28.4 \pm 9.9$ & $33.1 \pm 10.3$ & 0.492 \\
\hline Creatinine $(\mathrm{g} / \mathrm{dl})$ & $0.83 \pm 0.19$ & $0.79 \pm 0.17$ & 0.316 \\
\hline $\operatorname{AST}(\mathrm{U} / \mathrm{L})$ & $21.5(17-26.25)$ & $20.5(18.25-24)$ & 0.521 \\
\hline $\operatorname{ALT}(\mathrm{U} / \mathrm{L})$ & $19.5(13.75-24.25)$ & $18.5(15.5-23.5)$ & 0.613 \\
\hline Uric acid $(\mathrm{mg} / \mathrm{dl})$ & $4.92 \pm 1.0$ & $4.81 \pm 1.0$ & 0.766 \\
\hline Total bilirubin $(\mathrm{mg} / \mathrm{dl})$ & $0.6(0.4-0.9)$ & $0.7(0.5-0.8)$ & 0.697 \\
\hline $\mathrm{LDL}(\mathrm{mg} / \mathrm{dl})$ & $128.9 \pm 25.8$ & $134.9 \pm 33.7$ & 0.345 \\
\hline $\mathrm{HDL}(\mathrm{mg} / \mathrm{dl})$ & $42(34-48)$ & $44(36-52)$ & 0.328 \\
\hline Triglycerides $(\mathrm{mg} / \mathrm{dl})$ & $166.5 \pm 89.4$ & $169.3 \pm 81.7$ & 0.756 \\
\hline Total cholesterol $(\mathrm{mg} / \mathrm{dl})$ & $189.6 \pm 27.8$ & $201.8 \pm 41.6$ & 0.362 \\
\hline Hemoglobin $(\mathrm{g} / \mathrm{dl})$ & $13.9 \pm 1.6$ & $14.1 \pm 1.9$ & 0.817 \\
\hline White blood cell $\left(\times 10^{3} / \mathrm{dl}\right)$ & $7.9 \pm 1.8$ & $8.3 \pm 1.9$ & 0.325 \\
\hline Platelet $\left(\times 10^{3} / \mathrm{dl}\right)$ & $239.8 \pm 45.6$ & $243.1 \pm 51.6$ & 0.741 \\
\hline
\end{tabular}

MVA: microvascular angina; BMI: body mass index; SBP: systolic blood pressure; DBP: diastolic blood pressure; LVEF: left ventricular ejection fraction; LAD: left atrial diameter; LVEDD: left ventricular end-diastolic diameter; LVESD: left ventricular end-systolic diameter; IVST: interventricular septal thickness; PWT: posterior wall thickness; E: atrial E wave; A: atrial A wave; BUN: blood urea nitrogen; AST: aspartate aminotransferase; ALT: alanine aminotransferase; LDL: low-density lipoprotein; HDL: high-density lipoprotein

ventricle especially over the free wall and at the apex. Coronary arteries and their epicardial branches are embedded in the EAT [20]. Under physiological conditions, EAT functions in the maintenance of fatty acid homeostasis for the local vascular bed and has higher lipogenetic and lipolytic activity than other fat tissues [21]. Simultaneously, EAT is a well-known source of adipokines including a wide range of inflammatory mediators to a larger extent than the subcutaneous and omental fat [22] and secretes vasoactive products that 

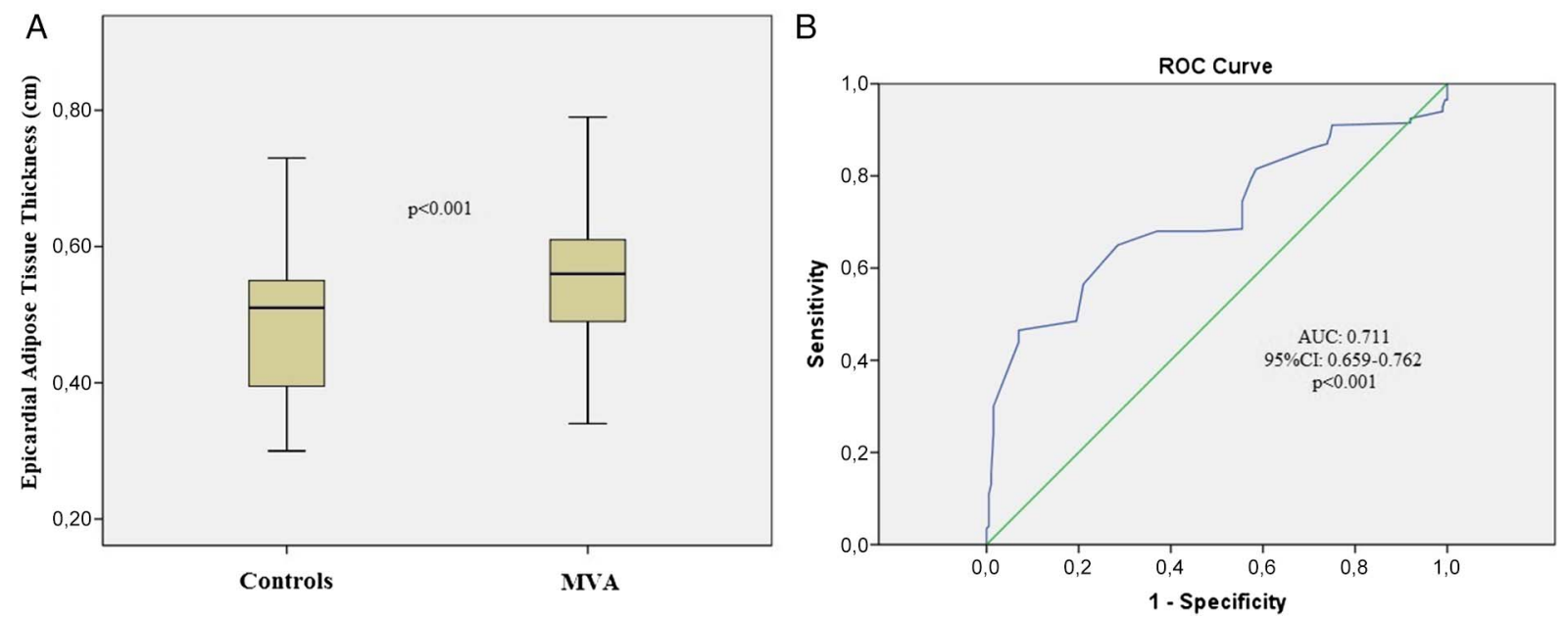

Fig. 2. (A) The box-plot graph comparing the epicardial adipose tissue thickness between patiens with microvascular angina (MVA) and the controls. (B) Receiver operating characteristic curve revealing the area under the curve for epicardial adipose tissue thickness to predict MVA

Table II Multivariate regression analysis showing independent predictors of microvascular angina

\begin{tabular}{lcccccc} 
& \multicolumn{3}{c}{ Univariate analysis } & \multicolumn{3}{c}{ Multivariate analysis } \\
& OR & $95 \%$ CI & $p$ value & OR & $95 \%$ CI & $p$ value \\
Age (years) & 1.015 & $0.991-1.039$ & 0.231 & 0.987 & $0.953-1.029$ & 0.671 \\
Gender: male & 0.885 & $0.595-1.315$ & 0.545 & 0.264 & $0.112-0.557$ & 0.103 \\
Hypertension & 0.751 & $0.489-1.154$ & 0.191 & 0.622 & $0.546-1.041$ & 0.691 \\
Diabetes mellitus & 0.851 & $0.517-1.401$ & 0.526 & 0.238 & $0.063-1.110$ & 0.171 \\
Dyslipidemia & 1.345 & $0.852-2.123$ & 0.203 & 0.783 & $0.345-1.229$ & 0.703 \\
Smoking & 0.896 & $0.566-1.418$ & 0.640 & 0.749 & $0.465-1.157$ & 0.243 \\
Increased EAT thickness & 1.327 & $1.012-1.897$ & $<0.001$ & 1.183 & $1.063-1.489$ & 0.023 \\
\hline
\end{tabular}

CI: confidence interval; OR: odds ratio; EAT: epicardial adipose tissue

regulate coronary arterial tone [23]. It has also been shown that periadventitial application of inflammatory mediators induces inflammatory cell influx into the arterial wall, coronary vasospasm, and intimal lesions [19]. Thus, inflammatory mediators secreted by the EAT surrounding coronary arteries may lead to impaired coronary blood flow reserve through an outside-to-inside cellular cross-talk. Lack of any fascia between the adipocytes and myocardium allows these direct interactions of vascular bed and the surrounding EAT [14]. Supported by the previous studies, increased EAT thickness may be associated with local and paracrine mechanisms that play role in the pathogenesis of MVA.

\section{Study limitations}

The primary limitation was that this study was a nonrandomized and single-center study with a relatively small number of patients. Second, coronary angiography was not performed in the control group; this issue is also open to criticism. A control group without symptoms and with a negative stress test and angiographically normal coronary arteries would have been ideal. However, it would be impossible to get approval from the ethics committee to perform coronary angiography in a patient group without symptoms and with a negative stress test in our country. Finally, magnetic resonance imaging (MRI), which was known as the gold standard for the measurement of EAT, was not used in this study. However, the widespread use of MRI is not practical for a screening parameter, and echocardiography has been validated against MRI for quantitative assessment of EAT [11].

\section{Conclusions}

The EAT thicknesses were observed significantly higher in MVA patients as compared to controls with similar cardiovascular risk factors. Increased EAT thickness may be associated with mechanisms that play a major role in the pathogenesis of MVA. Echocardiographic measurement of EAT thickness may be an additional and easy diagnostic tool for risk stratification of patients with 
typical chest pain. Those with an increased EAT may be particularly at risk for impaired coronary blood flow reserve, which is an early manifestation of atherosclerosis.

Funding sources: The authors received no financial support for the research, authorship, and/or publication of this article.

Authors' contribution: All of the authors contributed to planning, conduct, and reporting of the work. They had full access to all data in the study and take responsibility for the integrity of the data and the accuracy of the data analysis.

Conflict of interest: All the authors declare no conflict of interest.

\section{References}

1. Melikian N, De Bruyne B, Fearon WF, MacCarthy PA: The pathophysiology and clinical course of the normal coronary angina syndrome (cardiac syndrome X). Prog Cardiovasc Dis 50, 294-310 (2008)

2. Phan A, Shufelt C, Merz CN: Persistent chest pain and no obstructive coronary artery disease. JAMA 301, 1468-1474 (2009)

3. Singh M, Singh S, Arora R, Khosla S: Cardiac syndrome X: Current concepts. Int J Cardiol 142, 113-119 (2010)

4. Hurst T, Olson TH, Olson LE, Appleton CP: Cardiac syndrome X and endothelial dysfunction: New concepts in prognosis and treatment. Am J Med 119, 560-566 (2006)

5. Cannon RO: Microvascular angina and the continuing dilemma of chest pain with normal coronary angiograms. J Am Coll Cardiol 54, 877-885 (2009)

6. Chen C, Wei J, AlBadri A, Zarrini P, Bairey Merz CN: Coronary microvascular dysfunction - Epidemiology, pathogenesis, prognosis, diagnosis, risk factors and therapy. Circ J 81, 3-11 (2016)

7. Schejbal V: Epicardial fatty tissue of the right ventricle morphology, morphometry and functional significance. Pneumologie 43, 490-499 (1989)

8. Iozzo P: Myocardial, perivascular, and epicardial fat. Diabetes Care 34, S371-S379 (2011)

9. Iacobellis G, Willens HJ: Echocardiographic epicardial fat: A review of research and clinical applications. J Am Soc Echocardiogr 22, 1311-1319 (2009)

10. Friedewald WT, Levy RI, Fredrickson DS: Estimation of the concentration of low-density lipoprotein cholesterol in plasma, without use of the preparative ultracentrifuge. Clin Chem 18, 499-502 (1972)
11. Iacobellis G, Ribaudo MC, Assael F, Vecci E, Tiberti C, Zappaterreno A, Di Mario U, Leonetti F: Echocardiographic epicardial adipose tissue is related to anthropometric and clinical parameters of metabolic syndrome: A new indicator of cardiovascular risk. J Clin Endocrinol Metab 88, 5163-5168 (2003)

12. Lanza GA, Crea F: Primary coronary microvascular dysfunction clinical presentation, pathophysiology, and management. Circulation 121, 2317-2325 (2010)

13. Kemp HG: Left ventricular function in patients with the anginal syndrome and normal coronary arteriograms. Am J Cardiol 32, 375-376 (1973)

14. Iacobellis G, Corradi D, Sharma AM: Epicardial adipose tissue: Anatomic, biomolecular and clinical relationships with the heart. Nat Clin Pract Cardiovasc Med 2, 536-543 (2005)

15. Rosito GA, Massaro JM, Hoffmann U, Ruberg FL, Mahabadi AA, Vasan RS, O'Donnell CJ, Fox CS: Pericardial fat, visceral abdominal fat, cardiovascular disease risk factors, and vascular calcification in a community-based sample: The Framingham Heart Study. Circulation 117, 605-613 (2008)

16. Iacobellis G, Pellicelli AM, Sharma AM, Grisorio B, Barbarini G, Barbaro G: Relation of subepicardial adipose tissue to carotid intima-media thickness in patients with human immunodeficiency virus. Am J Cardiol 99, 1470-1472 (2007)

17. Mazurek T, Zhang L, Zalewski A, Mannion JD, Diehl JT, Arafat H, Sarov-Blat L, O'Brien S, Keiper EA, Johnson AG, Martin J, Goldstein BJ, Shi Y: Human epicardial adipose tissue is a source of inflammatory mediators. Circulation 108, 2460-2466 (2003)

18. Miyata K, Shimokawa H, Kandabashi T, Higo T, Morishige K, Eto Y, Egashira K, Kaibuchi K, Takeshita A: Rho-kinase is involved in macrophage-mediated formation of coronary vascular lesions in pigs in vivo. Arterioscler Thromb Vasc Biol 20, 2351-2358 (2000)

19. Shimokawa H, Ito A, Fukumoto Y, Kadokami T, Nakaike R, Sakata M, Takayanagi T, Egashira K, Takeshita A: Chronic treatment with interleukin-1 beta induces coronary intimal lesions and vasospastic responses in pigs in vivo. The role of platelet-derived growth factor. J Clin Invest 97, 769-776 (1996)

20. Rabkin SW: Epicardial fat: Properties, function and relationship to obesity. Obes Rev 8, 253-261 (2007)

21. Marchington JM, Mattacks CA, Pond CM: Adipose tissue in the mammalian heart and pericardium: Structure, foetal development and biochemical properties. Comp Biochem Physiol B 94, 225-232 (1989)

22. Engeli S, Schling P, Gorzelniak K, Boschmann M, Janke J, Ailhaud G, Teboul M, Massiéra F, Sharma AM: The adipose-tissue reninangiotensinaldosterone system: Role in the metabolic syndrome? Int J Biochem Cell Biol 35, 807-825 (2003)

23. Sacks HS, Fain JN: Human epicardial adipose tissue: A review. Am Heart J 153, 907-917 (2007) 